\title{
LAZOS INVISIBLES DE LA COMUNICACIÓN POLITICA. COMUNIDADES DE PARTIDOS POLÍTICOS EN TWITTER EN UNAS ELECCIONES MUNICIPALES
}

\author{
Invisible ties of political communication. \\ Communities of political parties on Twitter \\ in local government elections
}

Xabier Martínez-Rolán y Teresa Piñeiro-Otero

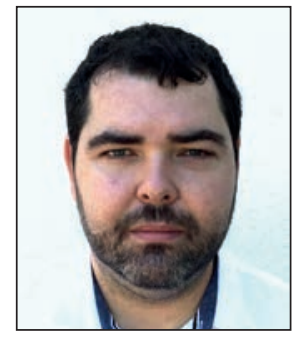

Xabier Martínez-Rolán es doctor en Comunicación por la Universidade de Vigo y profesor asociado en la Facultad de Ciencias Sociales y de la Comunicación en dicha universidad. Como gestor de comunidades online en el ámbito laboral, sus líneas de investigación se centran en el estudio de comunidades virtuales, uso y apropiación de redes sociales y nuevos formatos publicitarios, y fórmulas comunicativas en nuevos medios. http://orcid.org/0000-0002-7631-2292

Universidade de Vigo, Facultade de Ciencias Sociais e da Comunicación Campus A Xunqueira, s/n. 36005 Pontevedra, España xabier.rolan@uvigo.es

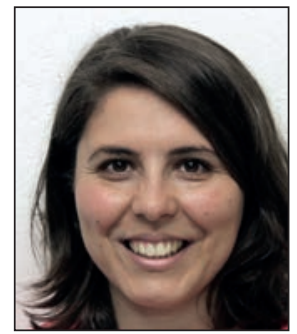

Teresa Piñeiro-Otero es doctora en Comunicación por la Universidade de Vigo y profesora contratada en la Universidade da Coruña, donde imparte docencia en el grado de Comunicación Audiovisual. Miembro del grupo de investigación Comunicación y Cultura Interactiva, ha orientado su investigación hacia las nuevas formas mediáticas en la era de la convergencia y a las nuevas manifestaciones de la comunicación política y el activismo en el contexto de la red social.

http://orcid.org/0000-0001-6414-2700

Universidade da Coruña, Facultade de Ciencias da Comunicación Campus de Elviña, s/n. 15071 A Coruña, España teresa.pineiro@udc.es

\section{Resumen}

La fuerte penetración de las tecnologías de la información y comunicación ha redefinido la interacción de los seres humanos. Internet como medio y las redes sociales como elementos disruptivos en la comunicación han obligado a todos los sujetos y estructuras políticas a adaptarse a un entorno digital con nuevas reglas para la interacción y participación colectiva. En este trabajo se analiza el entramado de relaciones que se tejen alrededor de los partidos políticos en un contexto electoral municipal. A partir de la teoría de grafos se han determinado las relaciones entre los agentes de la comunicación política en la twitteresfera. Se trata de una comunicación asimétrica que manifiesta, a través del grafo social de cada partido político, diferentes maneras de relacionarse con el electorado.

\section{Palabras clave}

Comunicación política; Twitter; Redes sociales; Medios sociales; Elecciones municipales; Partidos políticos; Medios de comunicación; Teoría de grafos; Gephi.

\section{Abstract}

The strong penetration of information and communication technologies have redefined the interaction of human beings. Internet as a medium and social networks as disruptive elements in communication have forced all subjects and political structures to adapt to a digital environment with new rules for interaction and collective participation. This paper analyzes the structure of relationships among political parties in a local government election. Based on graph theory, relationships between players in political communication in the twittersphere are analyzed. Using the social graph of each political party we found an asymmetric communication that reveals different ways of persuading the electorate.

\section{Keywords}

Political communication; Twitter; Social networks; Social media; Local elections; Political parties; Media; Graph theory; Gephi. 
Martínez-Rolán, Xabier; Piñeiro-Otero, Teresa (2017). "Lazos invisibles de la comunicación política. Comunidades de partidos políticos en Twitter en unas elecciones municipales". El profesional de la información, v. 26, n. 5, pp. 859-870.

https://doi.org/10.3145/epi.2017.sep.08

\section{Introducción}

La multiplicación de canales y modos de comunicación ha dado lugar a una nueva ecología mediática (Islas, 2015) que ha supuesto la pérdida del monopolio de la comunicación política para los actores políticos clásicos. En este contexto de autocomunicación de masas (Castells, 2009), la relevancia de un usuario en internet depende -más que de su influencia en la vida real- de los nodos de su red, de las interacciones que establece y su estructura.

Si bien en internet las relaciones "se producen de forma masiva y tienden a permanecer invisibles" (Del-Fresno-García, 2014 , p. 247), el estudio de la conversación política 2.0 puede determinar el valor de un perfil a partir de sus vínculos con los diversos agentes sociales, sus relaciones de entrada (menciones o interpelaciones al perfil político) y salida (redifusión de sus contenidos).

El análisis de las interacciones en medios sociales permite conocer la entidad de cada nodo a partir de un elevado volumen de conversaciones, constituyéndose como una fórmula de medición del impacto online de líderes y partidos desde la perspectiva de los receptores (Del-Fresno-García, 2014; Arcila-Calderón; Barbosa-Caro; Cabezuelo-Lorenzo, 2016). Frente a los posicionamientos teóricos que defienden la investigación 2.0 en torno a la centralidad del usuario, se propugna un enfoque centrado en el contexto (Tabak, 2014; González-Teruel, 2015).

Partiendo de la teoría de grafos, el presente estudio analiza la conversación política en Twitter en el marco de las elecciones municipales de 2015 con la finalidad de visibilizar las conexiones entre los actores políticos-sociales e identificar la estructura y patrones de comportamiento de las comunidades establecidas en torno a cada partido político.

Twitter se ha configurado como un canal fundamental para la comunicación política 2.0 (Ruiz-del-Olmo; Bustos-Díaz, 2015; Yoo; Gil-de-Zúñiga, 2014). Su naturaleza pública (no es necesario estar registrado para acceder a los contenidos), la brevedad de sus mensajes y su factor expansivo le dotan de gran visibilidad e influencia social; asimismo su elevada politización le confiere gran valor como termómetro de la opinión pública (Barberá; Rivero-Rodríguez, 2012; Kruikemeier, 2014; Peña-López; Congosto; Aragón, 2014; Martínez-Rolán; Piñeiro-Otero, 2016).

Las posibilidades de Twitter para la comunicación política han suscitado el interés de la academia dando lugar a numerosas aproximaciones, en su mayoría de carácter cuantitativo. Este enfoque ha orientado tanto los estudios pioneros en dicha plataforma (Java et al., 2007; Krishnamurthy; Gill; Arlitt, 2008; Huberman; Romero; Wu, 2009), como los análisis cibermétricos (Azorín-Richarte, 2012) o las clasificaciones de comunidades en la tweetesfera de Smith et al. (2014) que constituyen la base de este trabajo.
Pese a que la conversación política no constituye el objeto principal de Twitter, diversos estudios han señalado su valor para la movilización del electorado y la captación de indecisos (Calatrava, 2015). Como señala Calatrava (2016) la propia red de microblogging se autodefine como la mayor plataforma online de influencia política en España.

\section{La teoría de grafos en la investigación en medios sociales}

En los últimos años la investigación en medios sociales ha comenzado a superar la perspectiva cuantitativa para explorar otros aspectos a través de la teoría de grafos (Del-Fresno-García, 2014). Este enfoque funcionalista de la comunicación permite visibilizar las aristas (nexos de unión) que vertebran los nodos (actores de un grafo) en las comunidades virtuales.

Pese a su origen matemático, la teoría de grafos se ha elaborado en el marco de diversas disciplinas científicas alcanzando especial proyección en el análisis de las estructuras sociales y el desarrollo de la sociometría (Scott, 1991; Bezanilla; Miranda, 2012). El empleo de esta teoría en sociología cuenta con una extensa trayectoria desde el clásico de Galton (1907)

Las posibilidades de la teoría de grafos en la descripción del comportamiento de una red social, a partir de sus nodos y aristas, le confiere gran valor para el análisis de las interacciones 2.0. Su aplicación en Twitter ha llevado a Smith et al. (2014) a identificar seis arquetipos de conversación con sus propias estructuras de comunidad:

1) Multitudes polarizadas: habituales en la conversación política. Son grupos grandes y densos en los que los usuarios comparten su opinión, aunque apenas presenta conexión con los diferentes bloques.

2) Multitudes cercanas: conversaciones entre usuarios con un alto grado de conexión y escasa presencia de participantes aislados. El usuario de esta red comparte información con frecuencia y mantiene conversaciones con otros nodos.

3) Grupos de marcas: redes de baja densidad con participantes poco conectados entre sí. Los usuarios se agrupan en torno a marcas o personalidades a las que interpelan.

4) Grupos de comunidades: redes estructuradas en pequeños subgrupos con sus propias audiencias, influenciadores y fuentes de información. Dicha apariencia multimodal se asemeja a un mercado con puestos que funcionan como centros de actividad.

5) Redes de difusión: estructuras donde los usuarios siguen, difunden y comentan la actualidad diaria pero apenas establecen conversaciones con otros nodos. El centro de la red suele ser un medio de comunicación cuyo alcance es amplificado por su comunidad. 
6) Redes de apoyo: su interacción no se corresponde con una conversación sino con la gestión de quejas o sugerencias de los usuarios que se refleja en una estructura centro-radios opuesta a la red de difusión. Son habituales en los servicios de atención al cliente de empresas.

Inmersos en un contexto político de gran dinamismo y caracterizado por la aparición de fuerzas políticas de fuerte pujanza en el ámbito estatal con proyección local, resulta de interés conocer cómo se articula el discurso 2.0 de los principales partidos políticos en el marco de unas elecciones locales y la organización de los usuarios en tor-

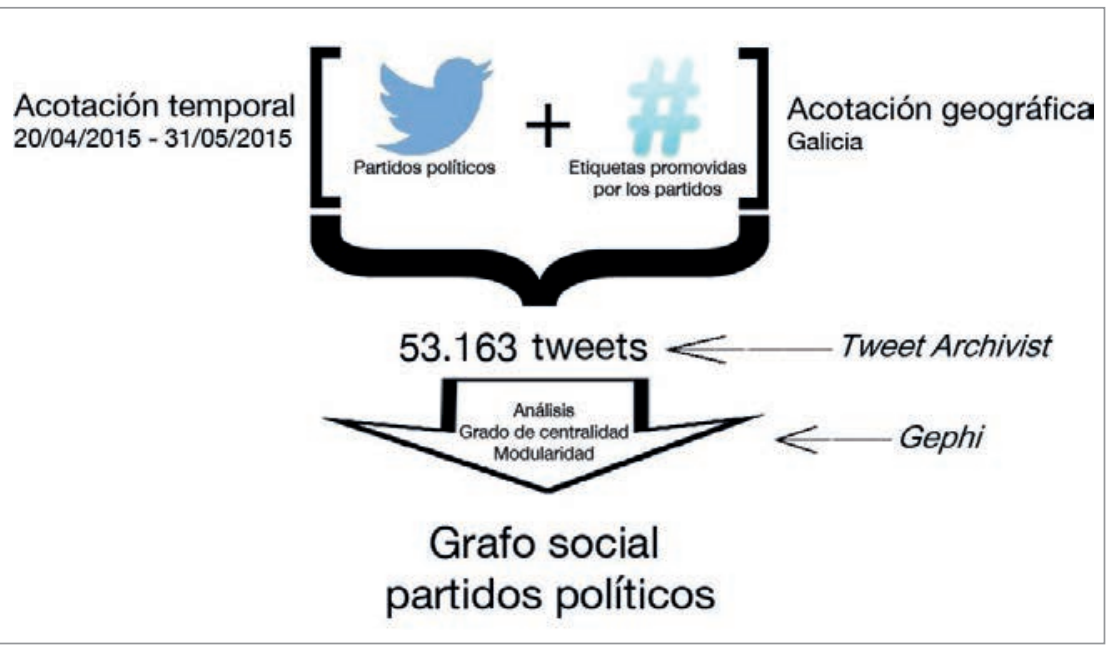

Gráfico 1. Acotación y herramientas metodológicas empleadas no a éste. Se parte de las siguientes hipótesis:

H1. Los nuevos partidos presentan una mayor adaptación a las plataformas sociales, que se manifiesta en el establecimiento de relaciones bidireccionales con los nodos de su red.

H2. Pese a la supuesta horizontalidad de las redes sociales, los medios de comunicación se erigen como actores relevantes en la conversación política.

\section{Materiales y métodos}

Para el estudio de una realidad tan porosa y dispersa como la comunicación política en Twitter, en el contexto de unas elecciones municipales (mayo 2015), se empleó el análisis de redes sociales como método de investigación. Este análisis se centró en dos atributos estructurales:

- grado medio de centralidad; contribución de un nodo según su ubicación en la red;

- modularidad de la red: fuerza de división de una red en agrupaciones o clusters.

A partir de la representación de la centralidad Eigenvector se pudo visibilizar la popularidad de los nodos, su relevancia e influencia, así como los puntos centrales de grupos cohesivos (Bonacich, 2007; Spizzirri, 2011).

De manera complementaria se han empleado otros atributos que Paniagua-López (2012) considera de valor para estudiar la actuación de las comunidades 2.0 como:
- diámetro de red: distancia máxima entre dos puntos conectados;

- longitud media del camino: distancia media entre todos los caminos posibles;

- densidad del grafo: volumen de conexiones de cada nodo respecto al total de conexiones posibles;

- grado medio del grafo: media de conexiones por nodo.

Dada la magnitud del fenómeno objeto de estudio se hizo una acotación múltiple del universo de análisis:

- Temporal: 20 de abril a 31 de mayo de 2015, período que abarca precampaña, campaña (8-22 de mayo), comicios (24 de mayo) y postcampaña electoral.

- Geográfica: Galicia como entorno regional que permitió a los partidos una comunicación mesosocial.

- Perfiles: principales partidos políticos nacionales y regionales con representación municipal u opciones de alcanzarla (Díez, 2015).

A partir de la muestra se implementó una observación no participante que permitió determinar los principales hashtags sobre los que se articuló la conversación política 2.0 durante el período analizado (estableciendo fases de 48 horas).

La captura con Tweet Archivist de las publicaciones emitidas con dichos hashtags, y que mencionasen a los partidos políticos, recabó un total de 53.163 tweets que fueron tratados con Gephi para su representación.

Tabla 1. Partidos políticos de la muestra de estudio.

\begin{tabular}{|c|c|c|c|}
\hline Partido político & Presencia & $\mathrm{N}^{\circ}$ candidaturas & Usuario en Twitter \\
\hline Partido Popular de Galicia (PPdeG) & Presencia municipal asentada & 314 & @PPdeGalicia \\
\hline Partido Socialista de Galicia (PSdeG) & Presencia municipal asentada & 304 & @PSdeG \\
\hline Bloque Nacionalista Galego (BNG) & Presencia municipal asentada & 264 & @OBloque \\
\hline Esquerda Unida $(E U)$ & Presencia municipal asentada & 14 & @EsquerdaUnida \\
\hline Ciudadanos Galicia (CsGalicia) & Perspectiva de alcanzar representatividad & 19 & $@$ @sGalicia \\
\hline Unión, Progreso y Democracia Galicia (UPyD Galicia) & Perspectiva de alcanzar representatividad & 9 & @UPyDGalicia \\
\hline Podemos Galicia & Perspectiva de alcanzar representatividad & 0 & @Podemos_Galicia \\
\hline
\end{tabular}


Este proceso permitió visibilizar la relevancia de los nodos -atendiendo a la redifusión de sus publicaciones- $y$ de las jerarquías establecidas en cada conversación social, dos aspectos clave para el análisis de la interacción política en Twitter (Medrano; Alonso-Berrocal; Figuerola, 2011).

\section{Organización de la conversación política $\mathbf{2 . 0}$}

El estudio sitúa los perfiles de partidos como epicentros de la conversación política sobre los que orbitan diversos clusters de usuarios. Pese al papel central de los partidos políticos en sus comunidades, éstas presentan diversas estructuras que son reflejo de las diferencias en la articulación del discurso, en las acciones y en el tipo de interacciones entre nodos. Unas divergencias que han permitido constatar la desigual representación

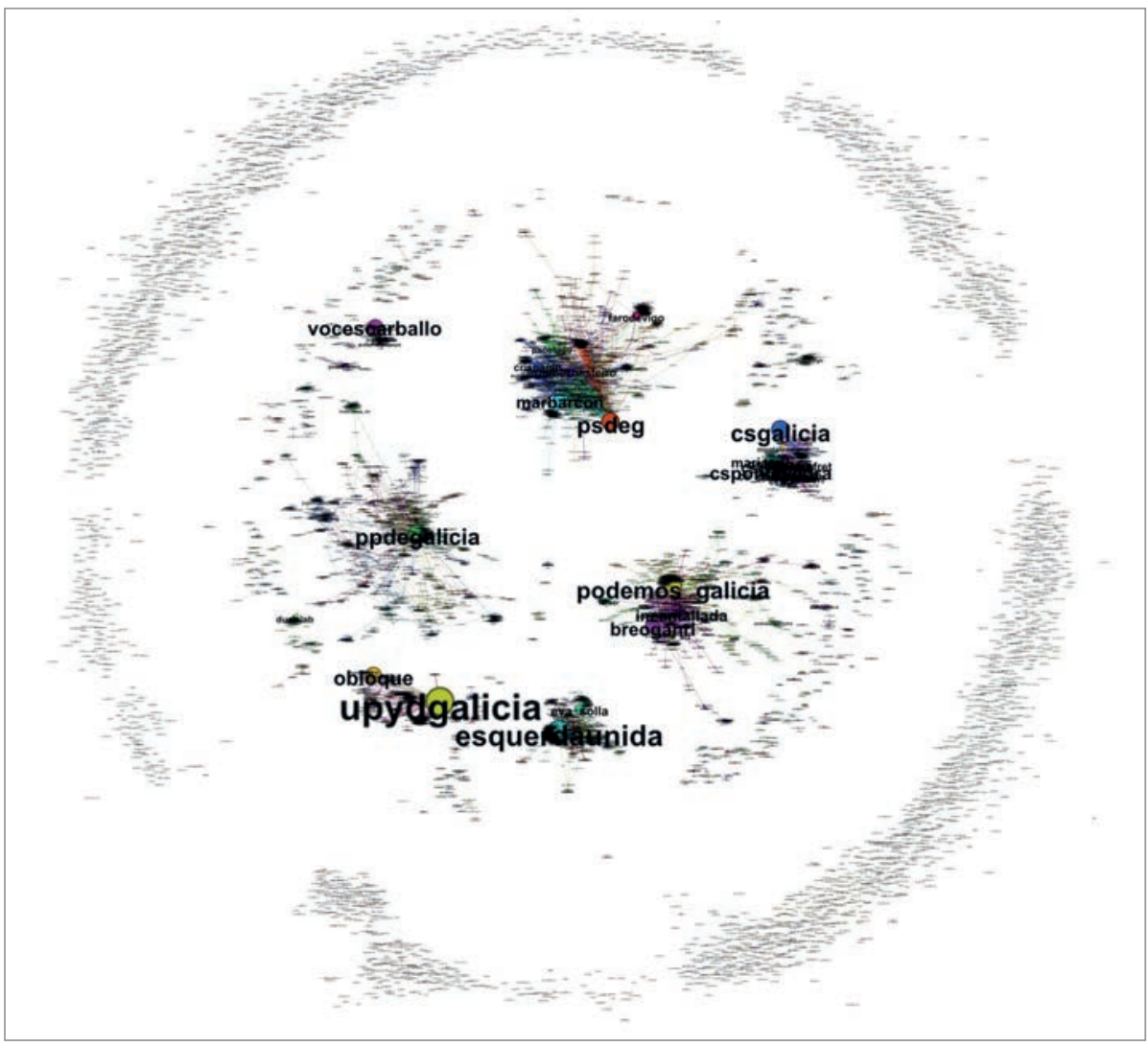
de la esfera política en Twitter.

La visualización conjunta de la conversación política 2.0 en la tweetesfera gallega revela la escasa relación entre las comunidades de los diferentes partidos, que parecen impermeables a la conversación política generada en los otros núcleos.

Hay una escasa relación entre las comunidades de los partidos, que parecen impermeables a la conversación política de los otros núcleos

Los grafos de cada comunidad reflejan una fuerte dependencia de los perfiles del partido y sus influenciadores, generando unas estructuras compactas que contrastan con el conjunto de usuarios -no articulados y escasamente interconectados- que participan de la conversación política 2.0 (cinturón exterior).
Dentro de esta conversación destacan UPyD y EU como los perfiles políticos de mayor redifusión (representados gráficamente con mayor tamaño), si bien son las cuentas de PSdeG, PPdeGalicia y Podemos Galicia (en adelante Podemos) quienes aglutinan las comunidades más fuertes y cohesionadas.

\subsection{Tipos de comunidades}

A partir del análisis de las relaciones de los perfiles de partidos con los nodos de su red, se pueden señalar tres tipos de comunidades:

- Estructuras fuertemente jerarquizadas (@UPyDGalicia, @EsquerdaUnida o @CsGalicia);

- Comunidades dispersas (@PSdeG, @PPdeGalicia o @PodemosGalicia);

- Redes heterárquicas (@OBloque).

Tabla 2. Principales atributos de los grafos de cada partido político. Elaborado a partir de Gephi

\begin{tabular}{|c|c|c|c|c|c|}
\hline Partido político & $\begin{array}{l}\text { Longitud } \\
\text { media }\end{array}$ & $\begin{array}{c}\text { Densidad del } \\
\text { grafo }\end{array}$ & $\begin{array}{l}\text { Diámetro } \\
\text { de la red }\end{array}$ & Grado medio & $\begin{array}{c}\text { Modularidad de } \\
\text { la red }\end{array}$ \\
\hline Partido Popular de Galicia (PPdeG) & 6,1 & 0,001 & 14 & 1,7 & 0,77 \\
\hline Partido Socialista de Galicia (PSdeG) & 3,1 & 0,001 & 8 & 1,9 & 0,54 \\
\hline Bloque Nacionalista Galego (BNG) & 2,4 & 0,042 & 7 & 8,5 & 0,52 \\
\hline Esquerda Unida $(E U)$ & 2,9 & 0,002 & 6 & 1,5 & 0,54 \\
\hline Ciudadanos Galicia (CsGalicia) & 3,1 & 0,004 & 9 & 1,6 & 0,54 \\
\hline Unión, Progreso y Democracia Galicia (UPyD Galicia) & 2,1 & 0,004 & 4 & 1,3 & 0,39 \\
\hline Podemos Galicia & 2,9 & 0,000 & 7 & 0,9 & 0,76 \\
\hline
\end{tabular}




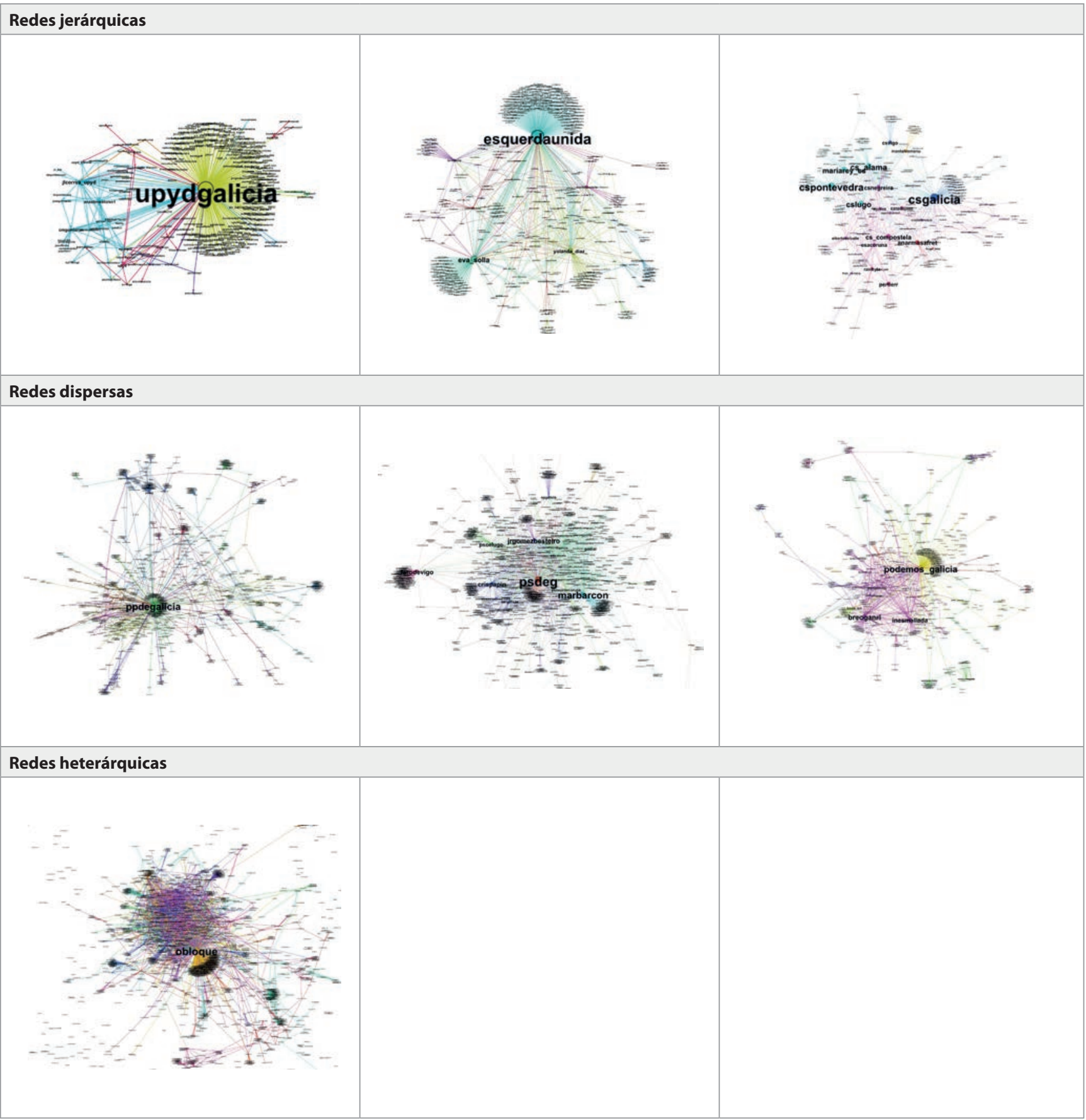

Estas configuraciones responden a la estrategia seguida por los partidos políticos en Twitter, así como la acción -organizada o fortuita- de otros perfiles dentro de su comunidad.

\subsubsection{Redes jerárquicas}

Presentan un flujo de información vertical dominado por las cuentas oficiales del partido, como sucede con las comunidades de @UPyDGalicia, @EsquerdaUnida o @CsGalicia.

El análisis de las interacciones en torno al perfil gallego de UPYD refleja una actividad reducida y fuertemente concentrada como refleja su diámetro de red, el más limitado de la muestra (4 puntos). Su grafo social revela el escaso asentamiento de la formación en la tweetesfera gallega: prácticamente la mitad de sus usuarios forman parte de la red estatal de $U P y D$ restando entidad a las cuentas regionales y locales.

La estructura presenta una longitud media muy baja $(2,1$ puntos) con tres polos que centralizan la actividad de la red:

- la cuenta matriz del partido y su secretaria general (@UPyD y @Rosadiezupyd);

- el perfil gallego del partido (@UPyDGalicia);

- un cluster más disperso que engloba a integrantes destacados del partido.

La comunidad de EU constituye un reflejo de su limitada presencia en la esfera política gallega y su integración en plataformas político-ciudadanas para las elecciones municipales. El grafo social de @EsquerdaUnida refleja su apoyo a otras 
fuerzas político-ciudadanas, como@ourenseencomun o @mareapontevedra, que constituyen clusters dentro de su red.

Pese a que la identidad de EU parece diluirse en el marco de dichas plataformas, el mapa de interacciones en torno a @EsquerdaUnida permite determinar una red jerárquica, dominada por los perfiles oficiales y líderes como@yolanda_diaz o @eva_solla, reflejo del apoyo directo y estructurado del partido.

El grafo social de @CsGalicia también señala una actividad agrupada y dirigida, domina-

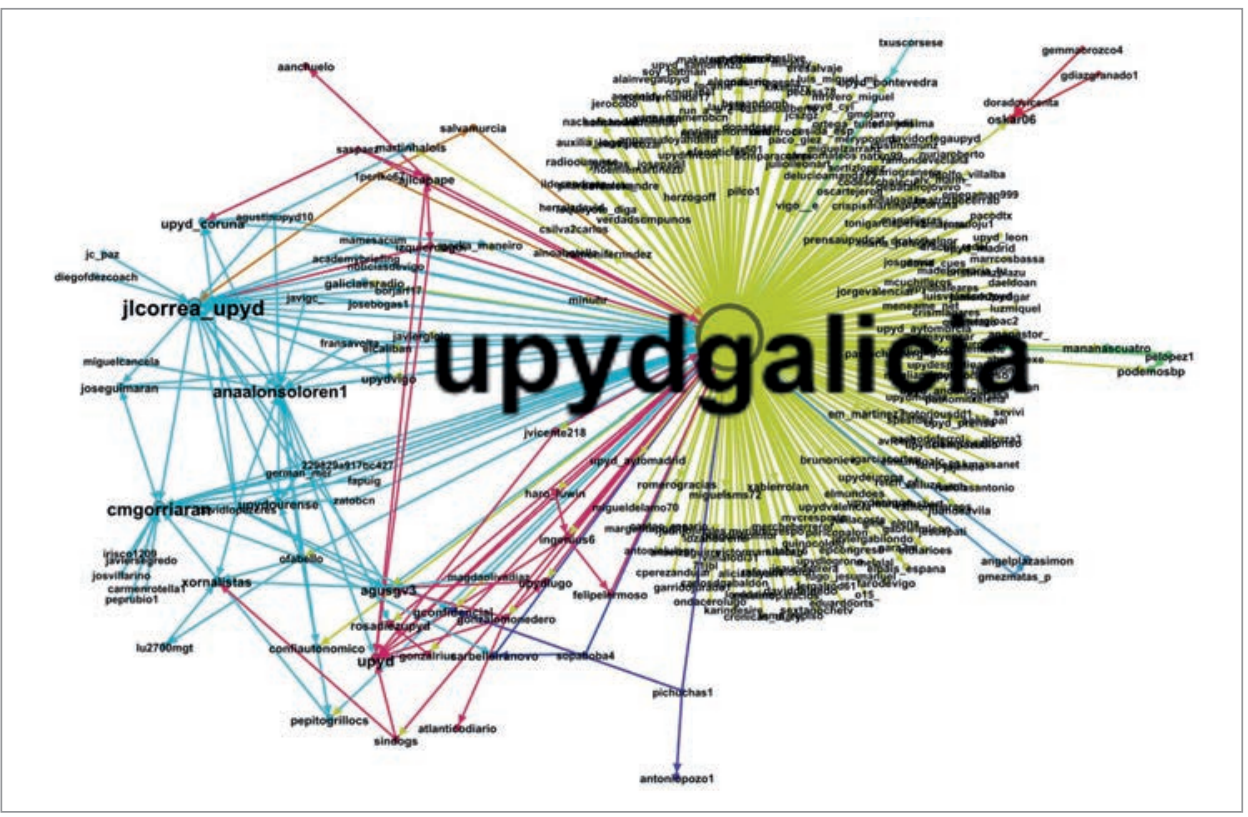
da por los perfiles autonómicos y locales en detrimento de los restantes usuarios. Dicha estructura refleja la institucionalización de la comunicación 2.0 de este partido que convierte a sus candidatos (@mariareycs, @canedojose, @rainbita, @pertierr) en portavoces de las líneas comunicativas del partido y epicentros de sus propios clusters.

Al igual que otras estructuras jerárquicas, la red de Ciudadanos en Twitter es poco compacta y -como reflejan la densidad de grafo $(0,004$ puntos), longitud media del camino $(3,128)$, diámetro (9 puntos), grado medio $(1,618$ puntos) o modularidad de la red $(0,54$ puntos)- presenta carencias estructurales.

\subsubsection{Redes dispersas}

Este tipo de redes se caracterizan por la descentralización de la información y el peso que adquieren otros colectivos y grupos de usuarios que interactúan con los perfiles de partidos políticos. El análisis efectuado ha permitido visibilizar este tipo de estructuras en las comunidades de @PSdeG, @PPdeGalicia y @ PodemosGalicia.

El perfil del PPdeG aglutina la mayor red de usuarios de la muestra (14 puntos de diámetro y 6 de longitud) con un mapa de interacciones rico y variado, aunque de poca densidad $(0,001$ puntos).

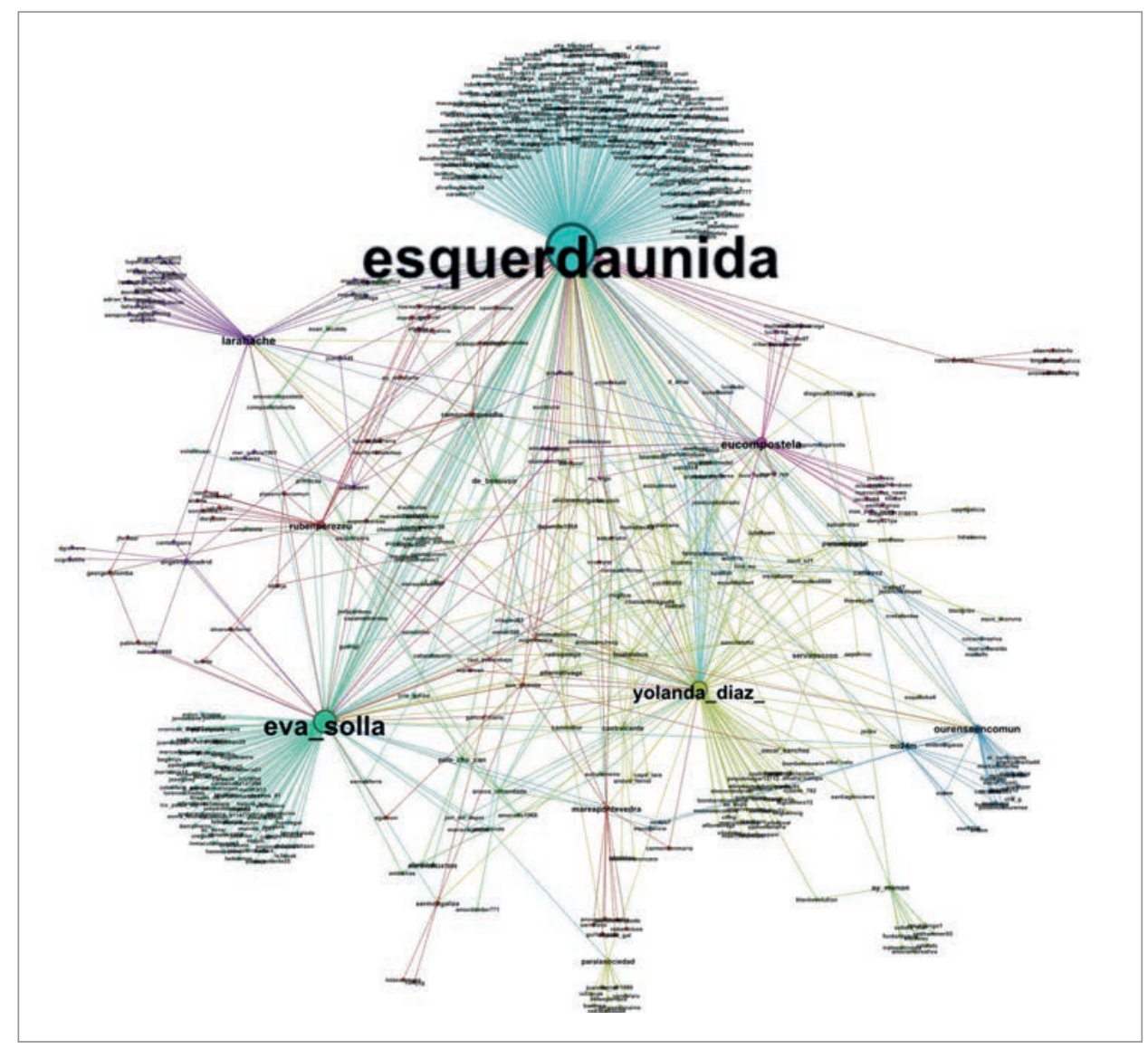

Gráfico 4. Grafo social de @EsquerdaUnida

Su carácter de partido hegemónico, tanto en los gobiernos locales gallegos como en los ejecutivos autonómico y central, dota al perfil popular de una imantación sobre otras fuerzas políticas, movimientos sociales y usuarios con independencia de su afiliación ideológica. Además de la presencia de cuentas de agrupaciones locales y personalidades del partido, reflejo de su asentamiento en la esfera política gallega, la comunidad de @PPdeGalicia se caracteriza por nodos que in- 


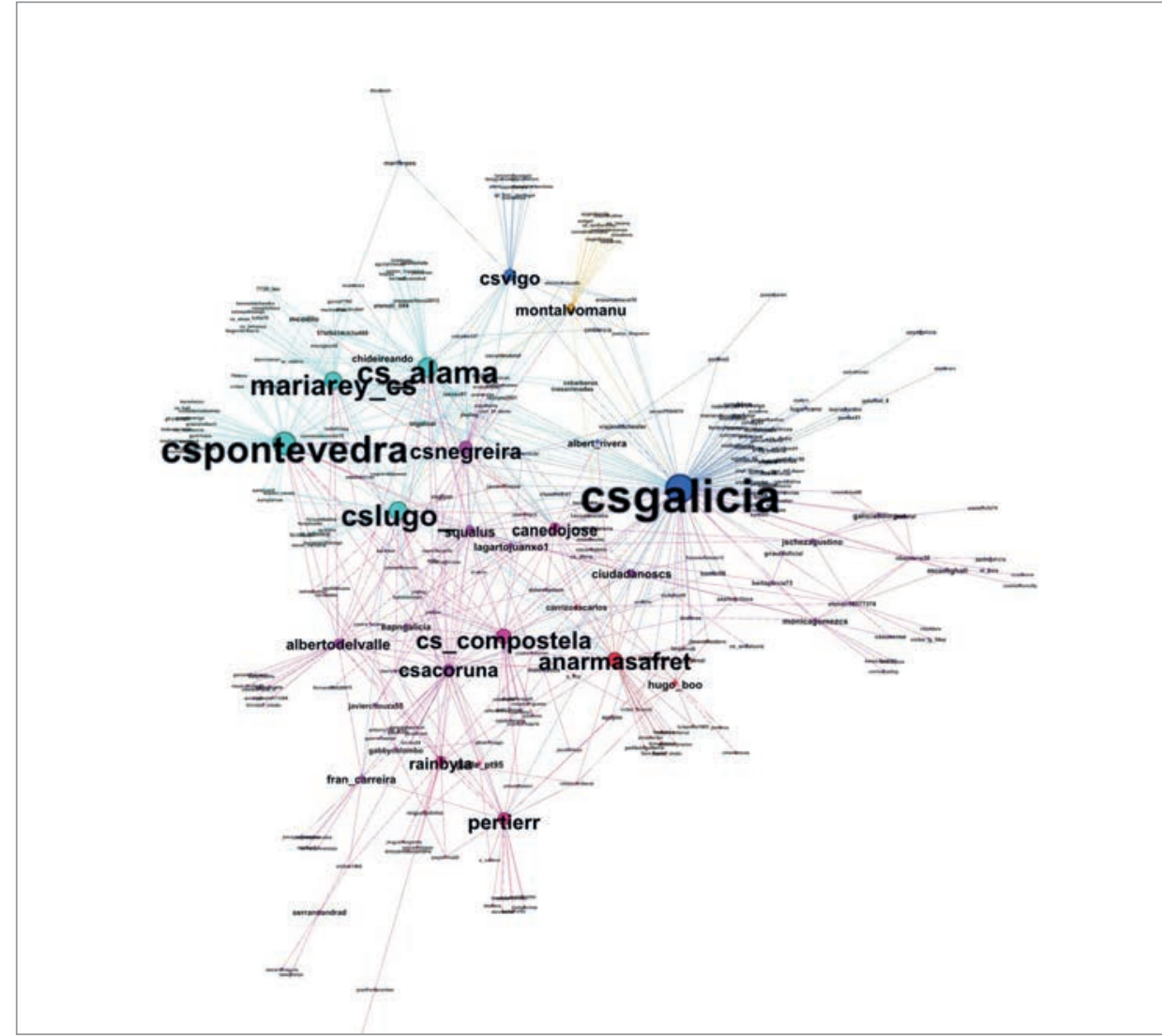

Gráfico 5. Grafo social de @CsGalicia

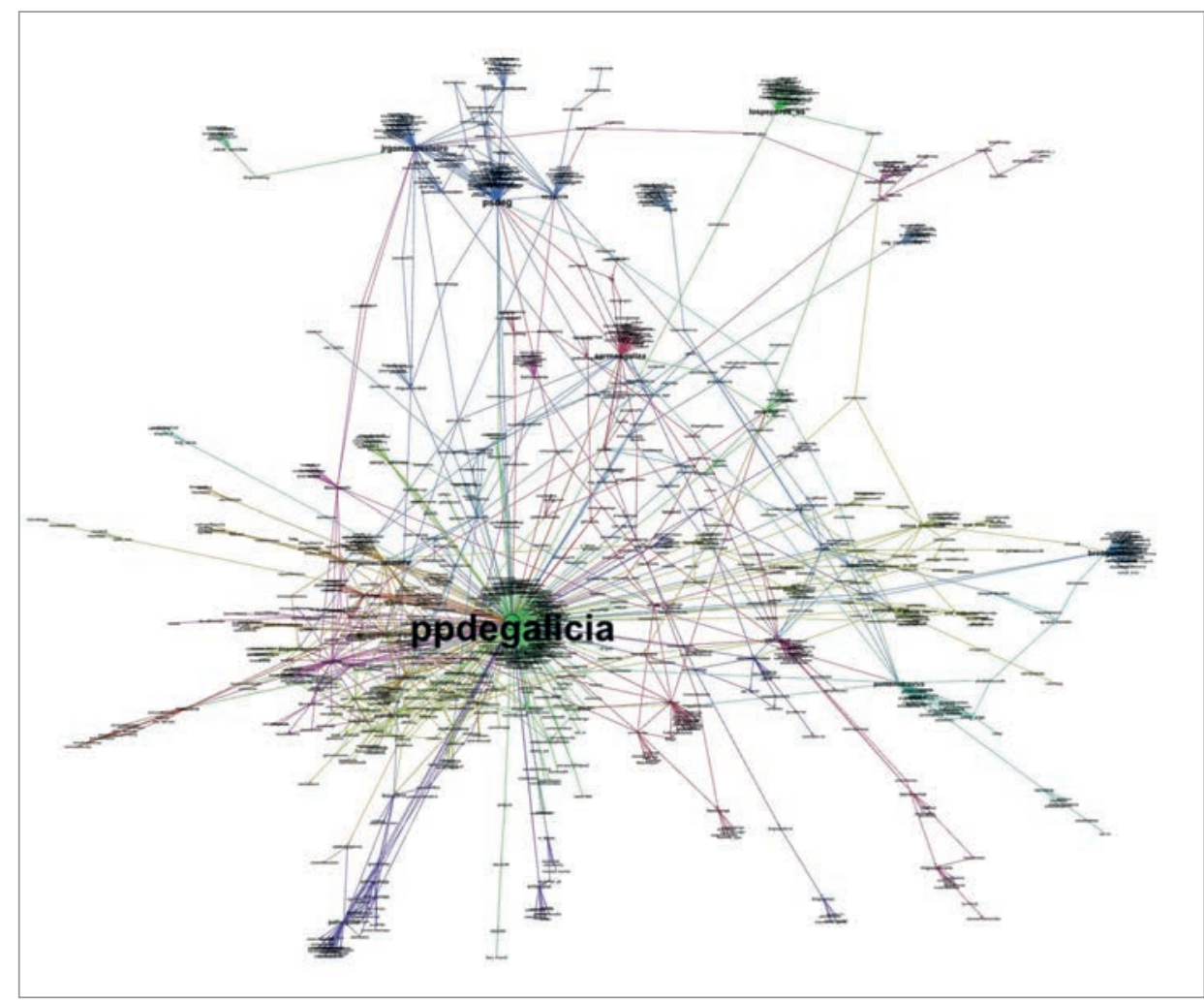

Gráfico 6. Grafo social de @PPdeGalicia teractúan por confrontación y clusters de usuarios liderados por candidatos, fuerzas políticas o movimientos sociales opuestos.

Esta particularidad se hace patente en la cohesión de la red, cuyo índice de modularidad $(0,77$ puntos) remite a una elevada cohesión entre los nodos de cada cluster (intra módulos) frente a la desconexión entre ellos (inter módulos).

El Partido Socialista de Galicia presenta en Twitter una comunidad de gran tamaño (8 puntos de diámetro), con diversos clusters de usuarios que forman una red bastante densa e interconectada $(0,54$ puntos de modularidad de red).

Las relaciones que se establecen en torno a @PSdeG son relativamente profundas $(3,1$ puntos de longitud media) aunque poco variadas.

Los principales nodos de esta comunidad son dirigentes del partido (@marbarcon o @jrgomezbesteiro), perfiles de agrupaciones locales y provinciales (@psoevigo o @psoelugo) y afiliados o usuarios muy comprometidos (@crispapin) con sus propias redes. Todos estos perfiles interactúan al abrigo de la cuenta matriz como refleja su diámetro (3 puntos), muy limitado para una red de este tamaño y densidad.

Aunque Podemos no presentó ninguna candidatura en las elecciones municipales de 2015, su peso en las encuestas y el apoyo a candidaturas político-ciudadanas (como $E U)$ propiciaron su inclusión en la muestra de estudio.

El grafo social de Podemos refleja la elevada participación de este perfil en la conversación política 2.0 que se articula a través de una estructura 
dispersa en torno a diversos clusters de usuarios.

La particularidad de esta comunidad radica en su elevado índice de modularidad $(0,76$ puntos) que subraya la sólida conexión interna de estos clusters, aunque con escasos vínculos entre módulos. Este elevado índice, solamente superado por la comunidad de @PPdeG, hace patente la polarización o uniformización de los usuarios en torno al perfil del partido.

La falta de una estructura consolidada de ámbito local se ha reflejado en la participación en la conversación política en torno a @Podemos Galicia de la cuenta matriz y principales líderes del partido. Este apoyo de la estructura estatal de Podemos ha sido menor que en otras formaciones de reciente creación como Ciudadanos o UPyD.

\subsubsection{Redes heterárquicas}

Esta red se caracteriza por contar con un flujo de información descentralizado que dibuja un grafo uniforme, en forma de malla, como sucede con la comunidad del BNG.

En torno al perfil de Twitter del BNG se articula una red compacta (7 puntos de diámetro), densa (longitud media de camino de 2,4 y densidad de 0,042 puntos) y de elevada conectividad (su grado medio de 8,5 puntos es el mayor de la muestra).

Esta particular estructura $y$, en concreto su densidad, podría relacionarse con el carácter regional de la formación que registra prácticamente toda su actividad en la esfera gallega (apenas se registran usuarios de fuera de Galicia).

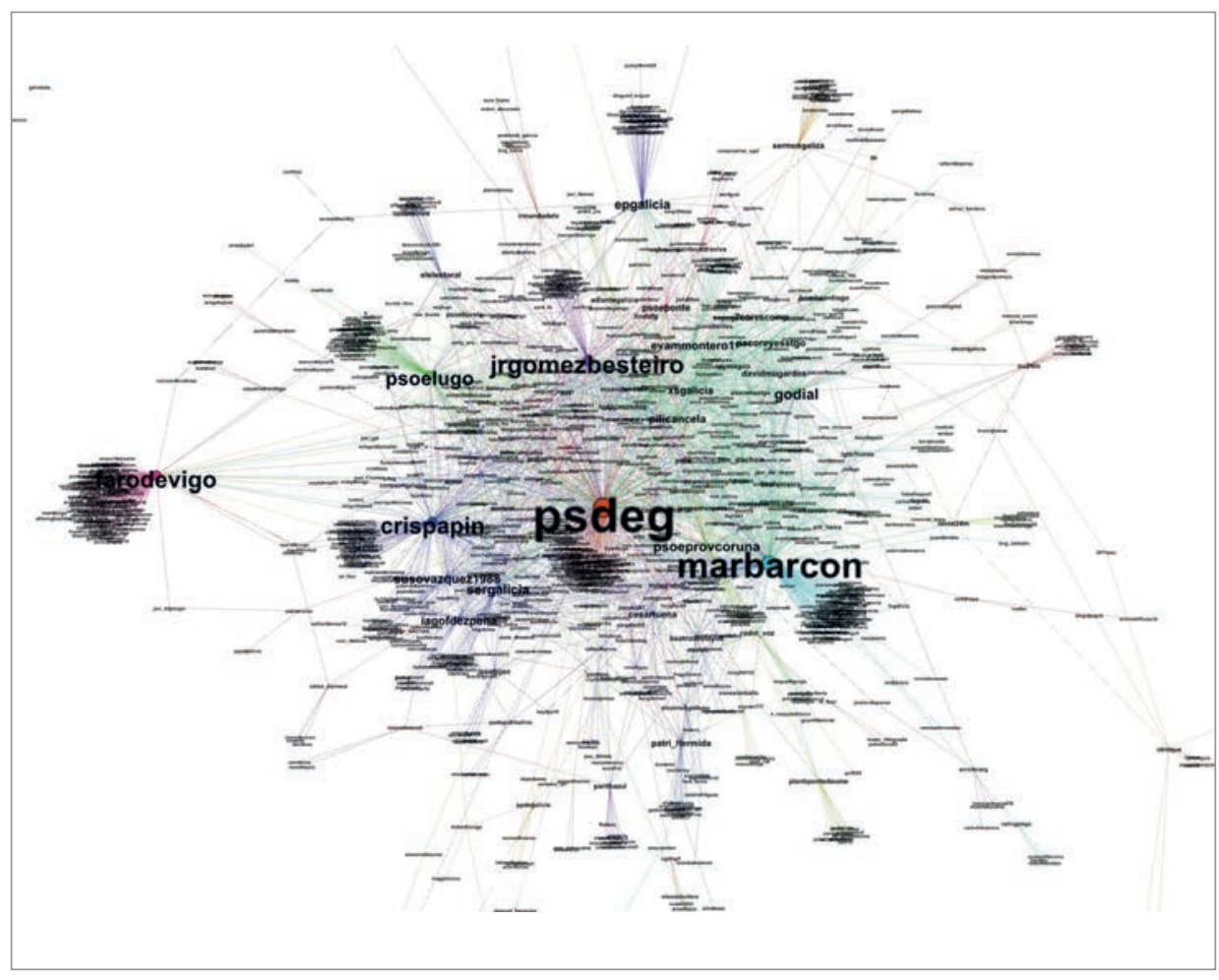

Gráfico 7. Grafo social de @PSdeG

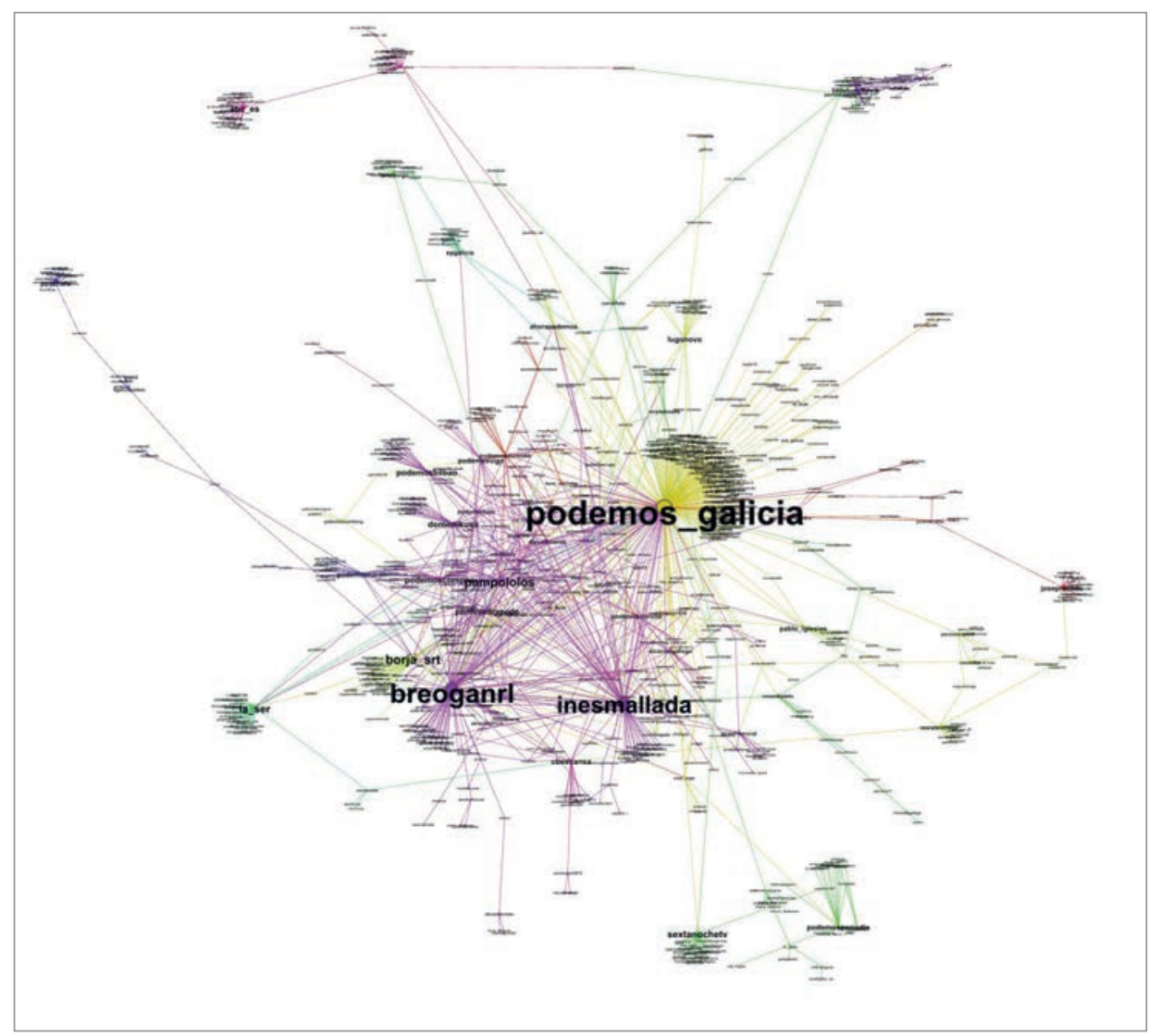

Gráfico 8. Grafo social de @PodemosGalicia 
Pese a la profundidad de esta red su grafo social no está articulado en torno al perfil del partido, sino que genera una trama profusa de usuarios que podrían funcionar al margen de la cuenta matriz. Así, aunque el grado máximo de centralidad se sitúa en @obloque, la estructura de nodos en torno a este perfil responde a una red interconectada distante de los patrones de unidireccionalidad o "aguja hipodérmica" de otros partidos con una organización jerárquica de la comunicación.

\subsection{Principales nodos}

Más allá del tipo de estructuras, el análisis de los grafos sociales también ha permitido determinar los principales nodos dentro de la red social de los partidos y que, a su vez, cuentan con sus propios clusters de usuarios.

\subsubsection{Perfiles políticos}

El carácter local de los comicios ha llevado a las diversas formaciones a involucrar a los perfiles de sus agrupaciones municipales, provinciales y regionales en su estrategia de comunicación 2.0. El análisis ha permitido señalar la aparición de líderes, candidatos o influenciadores (nacionales, autonómicos o locales) de cada partido entre los principales nodos de su red. Son unos usuarios (con sus propios clusters) que otorgan mayor visibilidad al discurso político del partido adaptándolo a cada contexto concreto.

Este tipo de perfiles emergen con fuerza en los grafos de estructuras fuertemente jerarquizadas. El peso que adquieren cuentas como @eva_solla, @Yolanda_diaz_, @eucompostela o @euourense en la red de EU; candidatos y formaciones locales como @mariareycs, @canedojose, @cspontevedra o @cslugo en la de CsGalicia o de la líder nacional @Rosadiezupyd y sus coordinadoras en la comunidad de UPyD Galicia pone de relevancia la fuerte institucionalización del discurso digital.

En las redes jerárquicas todos los influenciadores están directamente ligados al perfil del partido, lo que convierte a candidatos y cuentas locales en altavoces de las líneas comunicativas del partido y en líderes de sus propios clusters.

La excesiva dependencia de los perfiles oficiales y líderes en la comunicación de los partidos políticos en Twitter se hace también patente en redes distribuidas como la de Podemos, que sitúa en el centro de la interacción a @podemosgalicia y a un conjunto de subclusters directamente conectados con @breoganrl, perfil del secretario general autonómico (gráfico 8).

Otras redes dispersas como las de @PSdeG o @PPdeGalicia también articulan su discurso desde numerosas cuentas locales, candidatos o personalidades del partido, reflejo de su asentamiento local y la fortaleza de su estructura política. Estas cuentas suelen liderar clusters que integran otros nodos relevantes de la red. Un ejemplo es el del perfil del PSdeG compostelano, que acoge la actividad del candidato electoral (@pacoreysstgo) y de otros colectivos afines como la asociación LGTB@7corescomp.

No obstante, en el caso de algunas cuentas la limitada entidad de sus interacciones remite a su carácter instrumental, es decir, de redifusión de mensajes del partido, más que a su influencia real sobre un segmento de la esfera gallega. Así en la comunidad del @PPdeGalicia participan diversos dirigentes del partido (@jaimecastineira, @juanderuano, @manuelbaltar, @paulapra2 o @puypedro) con clusters débiles o inexistentes.

La representación gráfica de la conversación política 2.0 refleja una elevada polarización, en la que muchos usuarios interactúan para subrayar su adhesión o contraposición ideológica

La representación gráfica de la conversación política 2.0 refleja una elevada polarización de estas comunidades, donde muchos usuarios interactúan con el objetivo de subrayar su adhesión o contraposición ideológica. Destaca la comunidad del @PPdeG con una importante presencia de perfiles ideológicamente opuestos (@PSdeG, @podemosvigo, @ElBloque @EsquerdaUnida) o movimientos críticos (por ejemplo, la plataforma antidesahucios @pahvigotui) como nodos de su red (gráfico 6). 
Además de cuentas opuestas, el estudio también permite señalar la participación de militantes de una fuerza política en la red de partidos ideológicamente próximos. Este el caso de @pepitogrillocs, perfil afín a CsGalicia que participa activamente en la conversación social de @UPyDGalicia, probablemente con la intención de captar posibles electores.

En el caso de @OBloque su estructura heterárquica no permite determinar grupos de usuarios hiperconectados, si bien se hacen patentes las cuentas de líderes del partido (@xaviervence, @anamirandapaz) o influenciadores (@antonlosada).

Aunque los perfiles de medios de comunicación están presentes en todas las redes analizadas, tienen mayor relevancia en las comunidades de los partidos tradicionales

\subsubsection{Medios de comunicación}

El mapa de interacciones ha puesto de relevancia el peso de los medios de comunicación en la conversación política 2.0 en unas elecciones municipales.

Con independencia de su estructura y participación, todas las comunidades de partidos políticos presentan perfiles de medios de comunicación tradicionales ( $A B C, L a$ voz de Galicia, Cadena SER, etc.) o cibermedios nativos (Praza, Pontevedra viva, En Ferrol, Narón, etc.) como nodos de su red. Estos perfiles suelen conformar sus propios clusters de usuarios, algunos de ellos de gran relevancia dentro de la conversación política 2.0.

Aunque los perfiles de medios de comunicación están presentes en todas las redes analizadas, tienen mayor relevancia en las comunidades de los partidos tradicionales.

En el grafo social del @PSdeG medios tradicionales como Faro de Vigo, Cadena SER Galicia, Radio Voz o la agencia de noticias Europa Press Galicia se erigen como núcleos de poderosos clusters de usuarios no conectados con los principales nodos de la red. Del mismo modo, en la red del @PPdeGalicia destaca la presencia de un amplio y plural abanico de medios de comunicación -Sermos GalizaV Televisión, $A B C$ Galicia, Pontevedra viva o Europa Press Galicia- que refleja la polarización ideológica existente en torno a este partido.

La participación de los perfiles mediáticos en la conversación en torno a un determinado partido político en Twitter constituye una proyección de su relación en la vida real.

Los partidos políticos clásicos y mayoritarios cuentan con una mayor atención de los medios de comunicación (convencionales y cibermedios) que dan cobertura y actúan como potenciadores de su discurso también en la red.

Por su parte, las redes de partidos de reciente formación o minoritarios suelen integrar medios de comunicación de menor alcance o cibermedios nativos. Así, en la red de @EsquerdaUnida aparecen perfiles mediáticos como Porriño digital y cibermedios como Galicia confidencial o
El confidencial autonómico que participan de la conversación social en torno a @UPyDGalicia.

Finalmente resulta significativo destacar la limitada presencia de perfiles mediáticos en el grafo social del BNG.

\section{Conclusiones y discusión}

El estudio visibiliza el mapa de interacciones de la conversación política en el marco de las elecciones municipales de 2015 en Twitter. Los grafos sociales de los principales partidos permiten identifican tres tipos de redes atendiendo a su interacción con otros nodos:

- jerárquicas (UPyD Galicia, EU o Ciudadanos Galicia);

- dispersas (PPdeG y PSdeG);

- heterárquicas (BNG).

Siguiendo a Smith et al. (2014) dichas comunidades se corresponden con "multitudes polarizadas" a excepción de la red del BNG, más próxima al modelo de "multitud cercana", y la del PPdeG que podría clasificarse como "grupo de marca" por las interpelaciones de perfiles ideológicamente opuestos.

Dentro de estas comunidades adquieren especial relevancia las cuentas de agrupaciones locales o de personalidades del partido, que suelen liderar subredes de usuarios. También resulta destacable la presencia de perfiles mediáticos, de especial relevancia en las comunidades del PPdeG y PSdeG. Dichos perfiles reproducen el arquetipo de "red de difusión", cuya organización en subclusters de usuarios colisiona con la idea de internet como un espacio neutral, igual y plural: aunque Twitter brinda las mismas posibilidades técnico-creativas, no todos los usuarios cuentan con la misma amplificación e impacto de sus mensajes.

En cualquier caso, pese a la atracción que $P P d e G$ y PSdeG ejercen sobre el conjunto de perfiles mediáticos, se observa una correlación entre medios y partidos tradicionales, así como entre cibermedios nativos y formaciones políticas emergentes.

La articulación de la conversación política 2.0 en torno a comunidades independientes, vinculadas a los perfiles de partidos e impermeables a otros discursos, revela su excesiva polarización, en la línea señalada por Barberá y Rivero-Rodríguez (2012).

\section{Se observa una correlación entre medios y partidos tradicionales, así como entre cibermedios nativos y formaciones polí- ticas emergentes}

Solamente la comunidad de @PPdeGalicia cuenta con numerosos perfiles críticos y opositores; el papel hegemónico de este partido en los gobiernos municipales, autonómico y estatal lo convierten en objeto de las críticas de otras fuerzas políticas, plataformas y ciudadanía.

Asimismo se constata la ejecución de un filtro burbuja en la conversación social (Parisier, 2011) que dificulta la permeabilidad del discurso: los usuarios sólo escuchan lo que quieren y únicamente redifunden aquellos mensajes con los que están de acuerdo. 
En definitiva el estudio de la conversación política en torno a los perfiles de partidos en Twitter refleja una concepción de la comunicación 2.0 que todavía debe situarse en un estado de maduración.

Si bien, como señalan Martínez-Rolán y Piñeiro-Otero (2016), los partidos emergentes tienen en internet un aliado de excepción para llegar a los ciudadanos, los grafos sociales de @UPyDGalicia y @CsGalicia aún revelan una distribución jerárquica de la información. La ausencia de una estructura local consolidada ha llevado a dichos perfiles a elaborar una estrategia comunicativa convencional, no adaptada a la interactividad de la red, liderada por el perfil del partido con apoyos del aparato estatal.

Los usuarios sólo escuchan lo que quieren y únicamente redifunden aquellos mensajes con los que están de acuerdo

Por su parte la red de @PodemosGalicia muestra una mayor integración y participación de otros usuarios en la conversación política 2.0, reflejo de su carácter plural y su integración en plataformas político-ciudadanas.

En cualquier caso, sólo el BNG (partido de presencia consolidada) cuenta con una red distribuida adaptada a la esencia de la comunicación en plataformas sociales.

\section{Bibliografía}

Arcila-Calderón, Carlos; Barbosa-Caro, Eduar; Cabezuelo-Lorenzo, Francisco (2016). “Técnicas big data: análisis de textos a gran escala para la investigación científica y periodística". El profesional de la información, v. 25, n. 4, pp. 623-631.

https://doi.org/10.3145/epi.2016.jul.12

Azorín-Richarte, David (2012). Análisis cibermétrico de los contenidos de Twitter en España (2011). Universitat Politècnica de València.

http://riunet.upv.es/handle/10251/17326

Barberá, Pablo; Rivero-Rodríguez, Gonzalo (2012). “¿Un tweet, un voto? Desigualdad en la discusión política en Twitter". En: Congreso internacional en comunicación política y estrategias de campaña, Madrid, 6-7 julio 2012, pp. 200220. ISBN: 9788483197738

https://goo.gl/JnxVWz

Bezanilla, José-Manuel; Miranda, María-Amparo (2012). "La socionomía y el pensamiento de Jacobo Levy Moreno: una revisión teórica". Revista de psicología GEPU, v. 3, n. 1, pp. 148-180.

https://goo.gl/7DEx3v

Bonacich, Phillip (2007). "Some unique properties of Eigenvector centrality". Social networks, v. 29, n. 4, pp. 555-564. https://goo.gl/WVAXqg https://doi.org/10.1016/j.socnet.2007.04.002

Calatrava, Alfonso (2015). "Twitter, plataforma clave para movilizar electorado y captar indecisos". Blog Twitter, 13 mayo. https://blog.twitter.com/official/es_es/a/es/2015/twitterplataforma-clave-para-movilizar-electorado-y-captarindecisos.html

Calatrava, Alfonso (2016). "Los votantes españoles consideran que Twitter es la mayor plataforma de influencia política". Blog Twitter, 13 junio.

https://blog.twitter.com/official/es_es/a/es/2016/losvotantes-espa-oles-consideran-que-twitter-es-la-mayorplataforma-de-influencia-pol-tica.html

Castells, Manuel (2009). Comunicación y poder. Madrid: Alianza Editorial. ISBN: 9788420684994 https://goo.gl/rfivFW

Del-Fresno-García, Miguel (2014). "Haciendo visible lo invisible: visualización de la estructura de las relaciones en red en Twitter por medio del análisis de redes sociales". El profesional de la información, v. 23 n. 3, pp. 246-252.

https://doi.org/10.3145/epi.2014.may.04

Díez, Anabel (2015). "El PSOE se acerca al PP, Podemos se desploma y Ciudadanos se dispara". El país, 7 mayo.

http://politica.elpais.com/politica/2015/05/07/ actualidad/1430989220_519474.html

Galton, Francis (1907). “One vote, one value”. Nature, v. 75, p. 414.

http://www.nature.com/nature/journal/v75/n1948/ abs/075414a0.html

https://doi.org/10.1038/075414a0

González-Teruel, Aurora (2015). “Estrategias metodológicas para la investigación del usuario en los medios sociales: análisis de contenido, teoría fundamentada y análisis del discurso". El profesional de la información, v. 24, n. 3, pp. 321-328. https://doi.org/10.3145/epi.2015.may.12

Huberman, Bernardo; Romero, Daniel; Wu, Fang (2009). "Social networks that matter: Twitter under the microscope". First Monday, v. 14, n. 1.

https://doi.org/10.5210/fm.v14i1.2317

Islas, Octavio (2015). "La ecología de los medios: metadisciplina compleja y sistémica". Palabra clave, v. 18 n.4, pp. 1057-1083.

https://doi.org/10.5294/pacla.2015.18.4.5

Java, Akshay; Song, Xiaodan; Finin, Tim; Tseng, Belle (2007). "Why we twitter: Understanding microblogging usage and communities". En: $9^{\text {th }}$ WebKDD and $1^{\text {st }}$ SNA-KDD 2007 Workshop on web mining and social network analysis, San Jose (California), August 12, pp. 56-65. ISBN: 9781595938480 http://ebiquity.umbc.edu/_file_directory_/papers/369.pdf

Krishnamurthy, Balachander; Gill, Phillipa; Arlitt, Martin (2008). "A few chirps about Twitter". In: Procs of the First workshop on online social networks, Seattle, WA, USA, August 18, pp. 19-24. ISBN: 9781605581828

http://web2.research.att.com/export/sites/att_labs/ people/Krishnamurthy_Balachander/papers/twit.pdf https://doi.org/10.1145/1397735.1397741

Kruikemeier, Sanne (2014). "How political candidates use Twitter and the impact on votes". Computers in human behavior, v. 34, pp. 131-139. 
https://goo.gl/havGFQ

https://doi.org/10.1016/j.chb.2014.01.025

Martínez-Rolán, Xabier; Piñeiro-Otero, Teresa (2016). “Los memes en el discurso de los partidos políticos en Twitter: análisis del debate sobre el estado de la nación de 2015". Communication \& society, v. 29, n. 1, pp.145-160.

https://doi.org/10.15581/003.29.1.sp.145-160

Medrano, José-Federico; Alonso-Berrocal, José-Luis; Figuerola, Carlos (2011). Visualización de grafos web.

https://goo.gl/gLPUUn

Paniagua-López, Julián-Antonio (2012). Curso de análisis de redes sociales: metodología y estudio de casos. Granada: Editorial Universidad de Granada. ISBN: 9788433854735

Pariser, Eli (2011). The filter bubble. What the internet is hiding from you. New York: The Penguin Press. ISBN: 9781 594203008

Peña-López, Ismael; Congosto, Mariluz; Aragón, Pablo (2014). "Spanish Indignados and the evolution of the 15M movement on Twitter: Towards networked para-institutions". Journal of Spanish cultural studies, v. 15, n. 1-2, pp. 189-216. https://e-archivo.uc3m.es/handle/10016/21599 https://doi.org/10.1080/14636204.2014.931678

Ruiz-del-Olmo, Francisco-Javier; Bustos-Díaz, Javier (2016). "Del tweet a la fotografía, la evolución de la comunicación política en Twitter hacia la imagen. El caso del debate del estado de la nación en España" (2015). Revista latina de comunicación social, n. 71, pp. 108-123.

https://doi.org/10.4185/RLCS-2016-1086

Scott, John (1991). Social network analysis. Newbury Park, London: Sage. ISBN: 9780803984806

Smith, Marc; Rainie, Lee; Shneiderman, Ben; Himelboim, Itai (2014). "Mapping Twitter topic networks: From polarized crowds to community clusters". Pew Research Center. Internet \& technology, 20 Febr.

http://www.pewinternet.org/2014/02/20/mapping-twittertopic-networks-from-polarized-crowds-to-community-clusters

Spizzirri, Leo (2011). Justification and application of Eigenvector centrality.

https://sites.math.washington.edu/ morrow/336_11/ papers/leo.pdf

Tabak, Edin (2014). "Jumping between context and users: A difficulty in tracing information practices". Journal of the Association for Information Science and Technology, v. 65, n. 11, pp. 2223-2232.

https://goo.gl/nRCYn7

https://doi.org/10.1002/asi.23116

Yoo, Sung-Woo; Gil-de-Zúñiga, Homero (2014). “Connecting blog, Twitter and Facebook use with gaps in knowledge and participation". Communication \& society, v. 27, n. 4, pp. 33-48. https://doi.org/10.15581/003.27.4.33-48

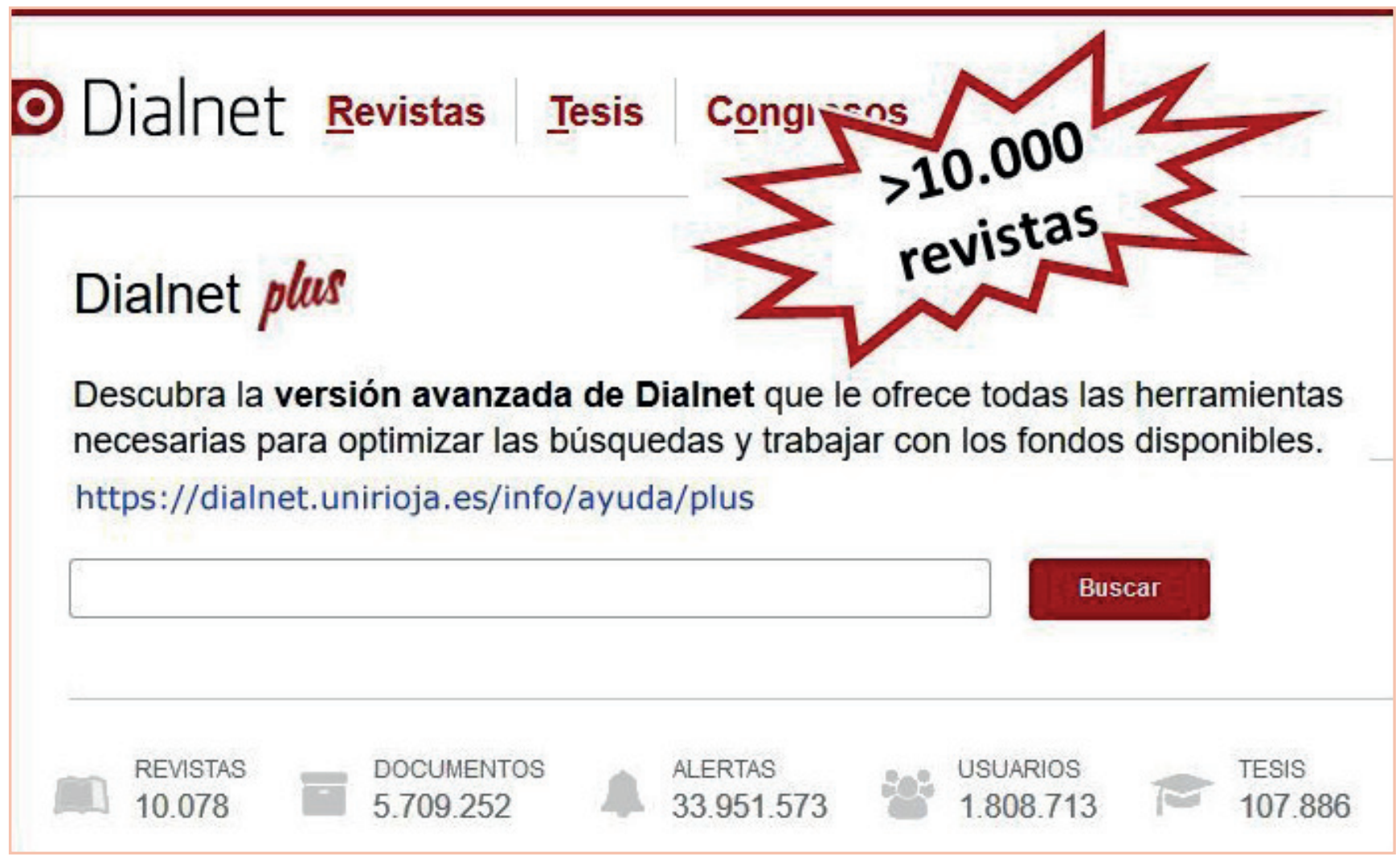

